\title{
Developmental potential of embryonic cells in a nucleocytoplasmic hybrid formed using a goldfish haploid nucleus and loach egg cytoplasm
}

\author{
TAKAFUMI FUJIMOTO*,1,2, TAIJU SAITO ${ }^{2, \#, ~ S U Z U ~ S A K A O ~}{ }^{1, \# \#, ~ K A T S U T O S H I ~ A R A I ~}{ }^{1}$ and ETSURO YAMAHA ${ }^{2}$ \\ ${ }^{1}$ Laboratory of Aquaculture Genetics and Genomics, Division of Marine Life Science, Graduate School of \\ Fisheries Sciences, Hokkaido University, Hakodate, Hokkaido, Japan, ${ }^{2}$ Nanae Fresh-Water Laboratory, Field \\ Science Center for Northern Biosphere, Hokkaido University, Nanae, Kameda, Hokkaido, Japan
}

\begin{abstract}
In teleosts, viable nucleocytoplasmic hybrids, formed by combining a nucleus from one species with the egg cytoplasm of another, have been used as one of the methods for breed improvement in aquaculture, but have been little exploited for developmental biology studies. Here, we used an artificial androgenesis technique to form nucleocytoplasmic hybrids comprising a goldfish haploid nucleus and loach egg cytoplasm. These hybrids were used to investigate interactions between the nucleus and cytoplasm during embryonic development. Additionally, the developmental characteristics of embryonic cells of nucleocytoplasmic hybrids were examined in chimeras produced by transplantation of blastomeres into recipient loach or goldfish embryos. We found that the nucleocytoplasmic hybrids arrested at the dome stage of embryonic development and did not form any gastrula structures. The goosecoid (gsc) and no tail (ntl) genes were expressed normally before gastrulation in nucleocytoplasmic hybrids, similar to diploid loach. However, expression of the gsc and $n t /$ genes was not maintained in nucleocytoplasmic hybrids. In chimeric embryos, blastomeres derived from nucleocytoplasmic hybrids were found to mix with the cells of recipient loach embryos at the gastrula stage. The transplanted blastomeres formed small clusters at the somitogenesis stage and, finally, small spots at the hatching stage. In contrast, when the blastomeres were transplanted into goldfish embryos, the transplanted blastomeres aggregated in the chimeric embryos. Thus, embryonic cells from nucleocytoplasmic hybrids that arrest before gastrulation could survive beyond the somitogenesis stage depending on the cytoplasmic environment in the recipient embryos.
\end{abstract}

KEY WORDS: nucleocytoplasmic hybrid, chimera, teleost, androgenesis

\section{Introduction}

A nucleocytoplasmic hybrid consists of the nucleus of one species and the cytoplasm of a different species. Such hybrids provide a valuable experimental model to examine interactions between the nucleus and cytoplasm during development. Nucleocytoplasmic hybrids are generally constructed by nuclear transplantation, although, in lower vertebrates, they can also be obtained by an induced androgenesis.

From the 1950 s to the 1970 s, various types of nucleocytoplas- mic hybrid were produced in amphibians using nuclear transplantation between different species, and their developmental capacities were examined (reviewed in Gallien, 1979). In general, intergeneric nucleocytoplasmic hybrids are able to develop to the late blastula or gastrula stage, but eventually die. However, intrageneric nucleocytoplasmic hybrids vary in their developmental capacities, depending on the species used as the nucleus

Abbreviations used in this paper: gsc, goosecoid; ntl, no tail.

\footnotetext{
*Address correspondence to: Takafumi Fujimoto. Laboratory of Aquaculture Genetics and Genomics, Division of Marine Life Science, Graduate School of Fisheries Sciences, Hokkaido University, 3-1-1 Minato-cho, Hakodate, Hokkaido 041-8611, Japan. Fax: +81-138-40-5537. e-mail: motchan@fish.hokudai.ac.jp Center for Elderly Nutrition and Development, Mukogawa Women's University, 6-46, Ikebiraki-cho, Nishinomiya, Hyogo 663-8558, Japan.
} 
donor and the egg cytoplasm recipient. Two types of developmental arrest were identified in incompatible nucleocytoplasmic hybrids: in the first, irreversible changes caused by chromosome aberrations occurred in the embryos until the blastula stage; in the second, some form of functional incompatibilities at the molecular level blocked further morphogenesis (reviewed in Gallien, 1979). The latter group had an intact genome that did not contain chromosome aberrations; if the nucleus was transplanted to an egg from its own species, a normal individual was obtained. These results indicate that the developmental capacities of nucleocytoplasmic hybrids depend on compatibility that is determined by the genetic distance between the donor and recipient species.

In teleosts, nucleocytoplasmic hybrids have been produced by transplanting a blastula nucleus into an enucleated egg using donor and recipient species that varied in their relatedness (Tung and Tung, 1963; Tung et al., 1973; Yan, 1989). Fertile nucleocytoplasmic hybrids resulting from a cell nucleus and cytoplasm from different genera or subfamilies were obtained in a series of such nuclear transplantations (Yan, 1989). These nucleocytoplasmic hybrids exhibit various phenotypic features: some of these depend on the nuclear donor, a few are intermediate, and others are influenced by cytoplasmic factors. Larvae that arrest in development at the larval stage were obtained from nucleocytoplasmic combinations between families, for example, between goldfish (Carassius auratus, family Cyprinidae, order Cypriniformes) and large-scale loach (Paramisgurnus dabryanus, family Cobitidae, order Cypriniformes) (Yan et al., 1990). In combinations between more distantly related species such as those in different orders, for example, between goldfish (Carassiusauratus, order Cypriniformes) and tilapia (Oreochromis nilotica, order Perciformes), however, embryonic development arrests at the blastula stage (Yan et al., 1990).

The alternative method for producing nucleocytoplasmic hybrids is an induction of androgenesis between different species. Androgenetic individuals are induced by fertilizing genetically inactivated (i.e. enucleated) eggs of one species with normal sperm of another species. These androgenesis-based nucleocytoplasmic hybrids can be used for regeneration or recovery of extinct or endangered fish genomes using cryopreserved sperm (Corley-Smith and Brandhorst, 1999). One particular problem for the latter application is the fact that the androgenotes have a haploid sperm-derived genome. Recovery of diploid status is generally achieved by inhibition of the first cleavage division. However, the rate of success of this process is low and, as a consequence, the survival rates of androgenotes are very low. Nevertheless, androgenesis has successfully produced adult nucleocytoplasmic hybrids, for example, intergeneric nucleocytoplasmic hybrids of goldfish nucleus and carp egg cytoplasm (Bercsényi et al., 1998), and interfamilial nucleocytoplasmic hybrids of mud loach Misgurnus mizolepis nucleus and carp egg cytoplasm (Nam et al., 2000, 2002).

As mentioned above, the results from studies using nuclear transplantation or induced androgenesis suggest that teleosts have a greater tolerance than amphibians in viability of nucleocytoplasmic hybrids formed between distantly related species. However, the problems of inviability and abnormal development of nucleocytoplasmic hybrids, which often appear in lethal combinations between distantly related species, have not received much attention from researchers and have been little investigated from the viewpoint of developmental biology. It is likely that nucleocytoplasmic interactions are more evident in lethal nucleocytoplasmic combinations than those in viable combinations, because nucleocytoplasmic incompatibilities will be expressed as failure of development in lethal combinations. Obviously, a stable induction of nucleocytoplasmic hybrids is a prerequisite for any analysis of interactions between nucleus and cytoplasm. Currently, there is clearly scope for improvement in the nuclear transplantation technique in teleosts since the frequency of nuclear transplants that achieve normal adulthood is very low even within the same species, for example, in medaka (Oryzias latipes) (Wakamatsu et al., 2001). Equally, stable induction of diploid androgenotes is much more difficult even in intraspecific combinations, because of the technical difficulty of inducing chromosome doubling using physical treatments (Komen and Thorgaard, 2007). One consequence of the very low rates of success for production of nucleocytoplasmic hybrids by either nuclear transplantation or induced diploid androgenesis, is the difficulty of observing their actual developmental potential and features due to the masking of results by the appearance of large numbers of unsuccessful embryos. In contrast, inducing haploid androgenesis between different species is relatively easy and the resultant androgenotes can be used as nucleocytoplasmic hybrids.

In the present study, we produced interfamilial nucleocytoplasmic hybrids by an artificial androgenesis technique using UVirradiated eggs of the loach (Misgurnus anguillicaudatus) and normal sperm of the goldfish (Carassius auratus). We then compared the developmental potential of these hybrids to those of embryos produced by normal fertilization of diploid loach, interspecific hybrid embryos (loach eggs $\times$ goldfish sperm), haploid gynogenesis (loach eggs $\times$ UV-irradiated goldfish sperm), haploid androgenesis (UV-irradiated loach eggs $\times$ loach sperm), and anuclear embryos (UV-irradiated loach eggs $\times$ UV-irradiated goldfish sperm). Additionally, we compared mesodermal gene expression in the goldfish haploid genome in loach cytoplasm to those in various controls. In order to determine the developmental potential of blastomeres derived from nucleocytoplasmic hybrids, we produced ten different types of chimeric embryo by transplantation of blastomeres from nucleocytoplasmic hybrids, diploid loach, diploid goldfish, or reciprocal hybrids between loach and goldfish into diploid loach or diploid goldfish embryos. We then compared the behavior of blastomeres of nucleocytoplasmic hybrids with that of blastomeres of the various types of the chimeric embryos in order to analyze effects of the nucleocytoplasmic interaction on a cell differentiation and an intercellular recognition.

\section{Results}

\section{Developmental potential of the nucleocytoplasmic hybrid}

All fertilized eggs produced in this experiment developed normally during the synchronous cleavage stages. After the dome stage, developmental differences became evident among the experimental groups. The hybrid (loach eggs $\times$ goldfish sperm) and the gynogenetic haploid loach embryos developed normally, similar to normal diploid loach embryos (Fig. 1A 1-4). However, germ ring formation and $100 \%$ epiboly were slightly delayed compared to normal diploid loach embryos (Fig. 1B 1-4 and 1C 14). In the androgenetic haploid loach embryos, development was 
further delayed during gastrulation (Fig. 1D1-4). Nevertheless, their blastoderms eventually covered the yolk cells and they reached the $100 \%$ epiboly stage. In contrast, when the nucleocytoplasmic hybrid embryos reached the dome stage, they did not form a dome-like shape similar to that seen in the diploid loach embryos (Fig. 1E1). At the germ ring stage and $75 \%$ epiboly stages, some nucleocytoplasmic hybrid embryos formed a domelike shape that resembled the diploid loach embryos at the dome stage (Fig. 1E2 and 1E3). The nucleocytoplasmic hybrid embryos did not show further epiboly or formation of the germ ring, but frequently arrested in embryogenesis at this dome-like stage at a time when the diploid loach embryos had reached $100 \%$ epiboly (Fig. 1E4). Furthermore, the shape of the blastoderm in the nucleocytoplasmic hybrid embryos became distorted and had a collapsed appearance. Anuclear embryos underwent cleavage but did not exhibit morphogenetic movement. These embryos arrested at the blastula stage (Fig. $1 \mathrm{~F} 1$ and 1F2), and, subsequently, their blastomeres fused and had a disrupted appearance (Fig. 1F3).

\section{gsc and ntl expression in nucleocyto- plasmic hybrids}

In normal diploid loach embryos, gsc and $n t /$ expression was detected from the blastula to gastrula stages, as reported previously (Fujimoto et al. 2004). gscwas expressed in the dorsal mesodermal region and $n t /$ was expressed in the panmesodermal region during gastrulation (Fig. 2A1-5). Here, we found that the expression patterns of these two genes were similar in hybrid (loach eggs $\times$ goldfish sperm), gynogenetic haploid, and androgenetic haploid loach embryos to those in the diploid loach embryos (Fig. 2B 1-5, C 1-5 and D 1-5). However, expression of the genes was delayed compared to diploid loach embryos due to their retarded development. There was no indication that either $g s c$ or $n t /$ was expressed in anuclear embryos at any stage of their development (Fig. 2F 1-4).

In nucleocytoplasmic hybrid embryos, $g s c$ and $n t /$ showed normal expression at the dome stage (Fig. 2E1 and 2E2). At the time when diploid loach embryos had reached the germ ring stage, the genes were expressed in nucleocytoplasmic hybrid embryos, but the staining intensities were weaker than those observed in other embryo types, except for anuclear embryos (Fig. 2E3). At the time when diploid loach embryos were at the $75 \%$ epiboly, gsc expression in nucleocytoplasmic hybrids was maintained in the blastoderm, which did not migrate to the animal pole. $n t /$ expression at the marginal blastoderm
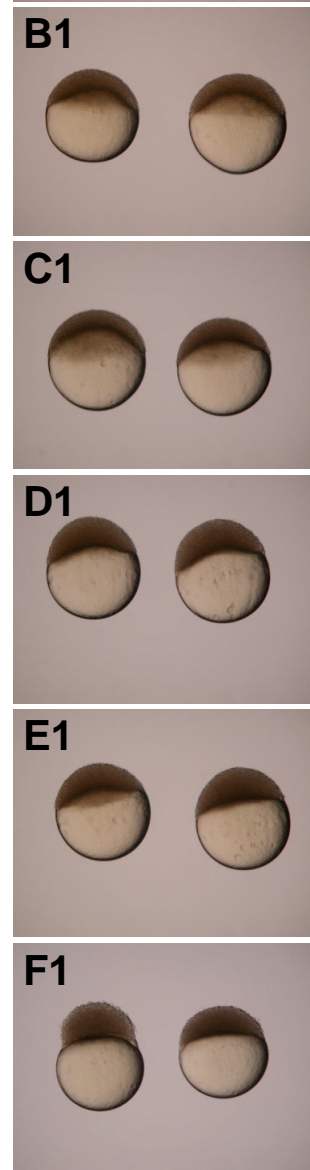
indicates $1 \mathrm{~mm}$. almost disappeared (Fig. 2E4). Finally, expression of the genes was not detectable in the blastoderm of nucleocytoplasmic hybrids at the time when diploid loach embryos were at the $100 \%$ epiboly stage (Fig. 2E5).

\section{Behavior of donor cells from nucleocytoplasmic hybrids in chimeric embryos}

Blastomeres from lethal nucleocytoplasmic hybrids were transplanted into diploid loach and diploid goldfish embryos and the distribution patterns of these cells were traced at the gastrula, somitogenesis and hatching stages. In addition, the behavior of donor cells from nucleocytoplasmic hybrids transplanted into two different recipients was compared with control chimeras consisting of various combinations of donor cells and recipient embryos.
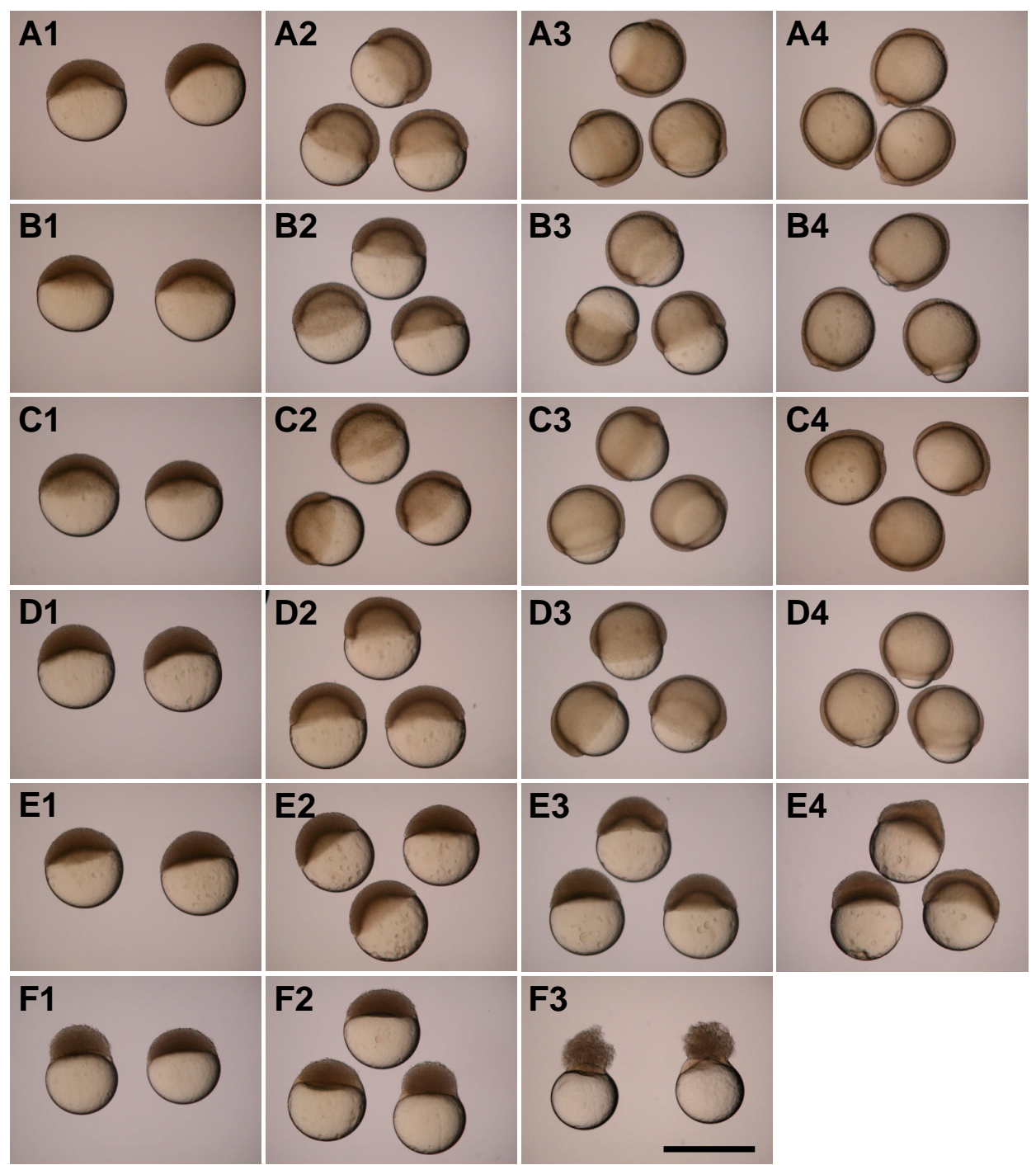

Fig. 1. Embryonic development during gastrula period in the six experimental groups. (A) Diploid loach embryos. (B) Hybrid embryos (loach eggs xgoldfish sperm). (C) Gynogenetic haploid loach embryos (loach eggs $\times$ UV goldfish sperm). (D) Androgenetic haploid loach embryos (UV loach eggs xloach sperm). (E) Nucleocytoplasmic hybrid embryos (UV loach eggs $\times$ goldfish sperm). (F) Anuclear embryos (UV loach eggs $\times$ UV goldfish sperm). Column 1, the dome stage. Column 2, the germ ring stage. Column 3, the $75 \%$ epiboly stage. Column 4 , the $100 \%$ epiboly stage. Scale bar 
When diploid loach embryos were used as recipients, donor cells from diploid loach or hybrid (loach eggs $\times$ goldfish sperm) embryos with loach cytoplasm were distributed in a scattered fashion and intermingled with the recipient cells in gastrula stage chimeric embryos (Fig. 3A1 and 3B1). Subsequently, the donor cells were distributed throughout the chimeric embryos at the somitogenesis stage and differentiated into various organs at the hatching stage (Fig. 3A 2-3 and 3B2-3). Donor cells from nucleocytoplasmic hybrid embryos likewise showed a scattered distribution and were intermingled with recipient cells in diploid loach embryos at the gastrula stage (Fig. 3C1). However, at the somitogenesis stage, the donor cells formed a number of small clusters in various parts of the chimeric embryos (Fig. 3C2). A large proportion of these clustered donor cells were not likely to be incorporated to embryogenesis, whereas donor cells from diploid loach and hybrid with loach cytoplasm were distributed at various parts of embryo and normally differentiated into various tissues (Fig. 3A 2-3 and 3B 2-3). Then, donor cells from the nucleocytoplasmic hybrid were present as small, scattered spots in hatching larvae (Fig. 3C3). Furthermore, the number of cells decreased after the somitogenesis stage. In contrast, donor cells derived from diploid goldfish and hybrid (goldfish eggs $\times$ loach sperm) embryos with goldfish cytoplasm aggregated at the gastrula stage to form a large cluster of donor cells (Fig. 3D1 and $3 E 1)$. Subsequently, the donor cells formed several smaller clusters at the somitogenesis stage that were maintained into the hatching stage (Fig. 3D 2-3 and 3E 2-3).

When diploid goldfish embryos were used as recipients, donor cells from diploid loach or hybrid (loach eggs $\times$ goldfish sperm) embryos with loach cytoplasm formed large clusters during embryogenesis (Fig. 4A 1-3 and 4B 1-3). In diploid goldfish recipients, donor cells derived from nucleocytoplasmic hybrids also formed large clusters at the gastrula and somitogenesis stages (Fig. 4C 1-2). However, the clusters changed to form small spots in a restricted part of the recipient hatching larvae (Fig. 4C3). In contrast, donor cells from diploid goldfish or hybrid (goldfish eggs $\times$ loach sperm) embryos with goldfish cytoplasm intermingled with recipient cells at the gastrula stage and were present in various parts of the recipient embryos at the somitogenesis and hatching stages (Fig. 4D 1-3 and 4E 1-3).

\section{Discussion}

Interspecific androgenetic embryos comprising goldfish haploid nuclei and loach cytoplasm are regarded as nucleocytoplasmic hybrids and can thus be used to analyze embryogenesis with respect to aspects of the developmental process and gene expression. The use of these hybrid embryos raises the question of whether the developmental process in a haploid nucleocytoplasmic hybrid is truly equivalent to those of diploid embryos. From the observations reported in a previous study, it is clear that the developmental potential of a haploid embryo is distinct from that of a diploid embryo, since
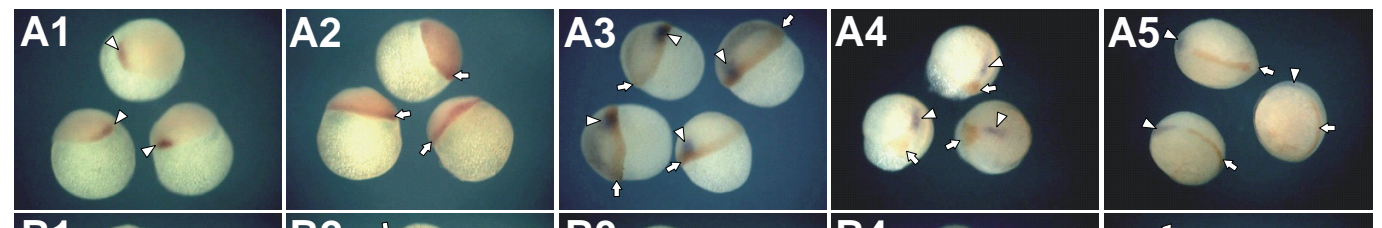
the haploid embryos frequently exhibited abnormal morphology, the so-called haploid syndrome, and died before or soon after hatching (Fujimoto et al., 2007). An earlier study reported that the development process in haploid nucleocytoplasmic hybrids was poorer than in intraspecific haploids in amphibians (Hennen, 1965). Such a
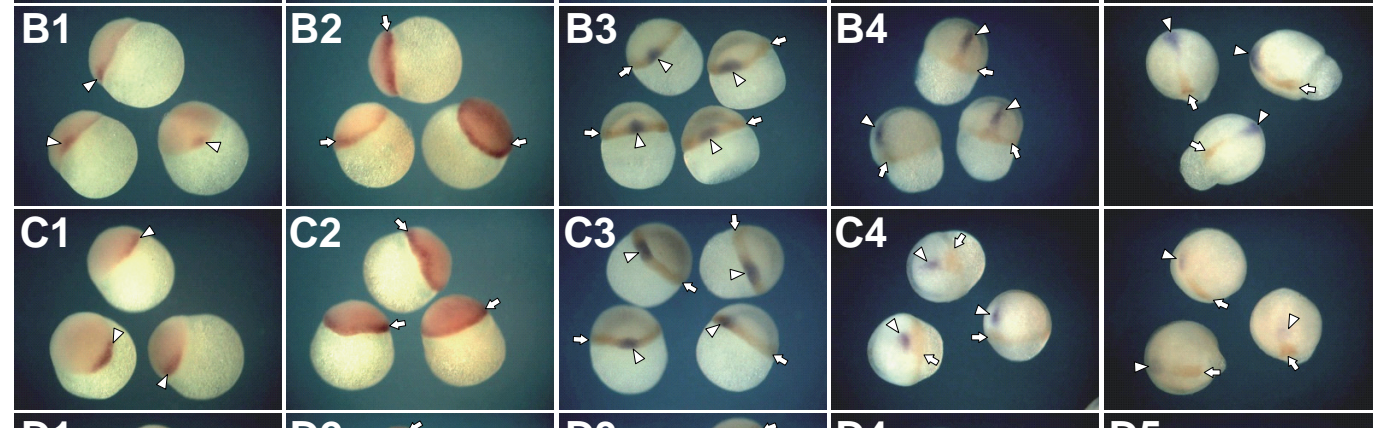

Fig. 2. gsc and $n t l$ expression detected by whole mount in situ hybridization during epiboly. (A) Diploid loach embryos. (B) Hybrid embryos (loach eggs xgoldfish sperm). (C) Gynogenetic haploid loach embryos (loach eggs $\times$ UV goldfish sperm). (D) Androgenetic haploid loach embryos (UV loach eggs $\times$ loach sperm). (E) Nucleocytoplasmic hybrid embryos (UV Ioach eggs $\times$ goldfish sperm). (F) Anuclear embryos (UV loach eggs $\times$ UV goldfish sperm). Column 1, the dome stage; gsc expression. Column 2, the dome stage; ntl expression. Column 3, the germ ring
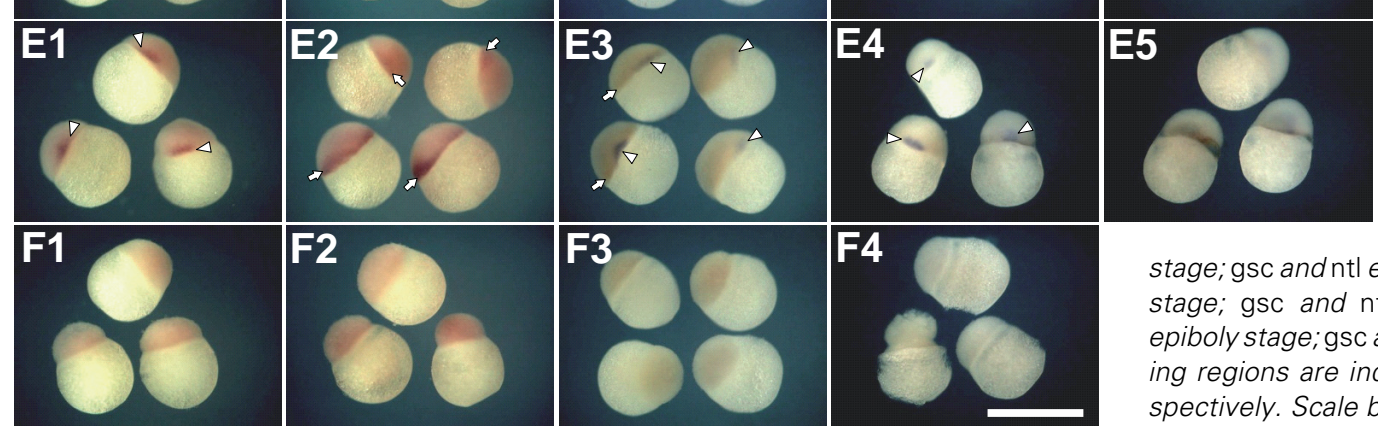

stage; gsc and ntl expression. Column 4, the $75 \%$ epiboly stage; gsc and $\mathrm{ntl}$ expression. Column 5, the 100\% epiboly stage; gsc and ntl expression. gsc and ntl expressing regions are indicated in arrowheads and allows, respectively. Scale bar indicates $1 \mathrm{~mm}$. 

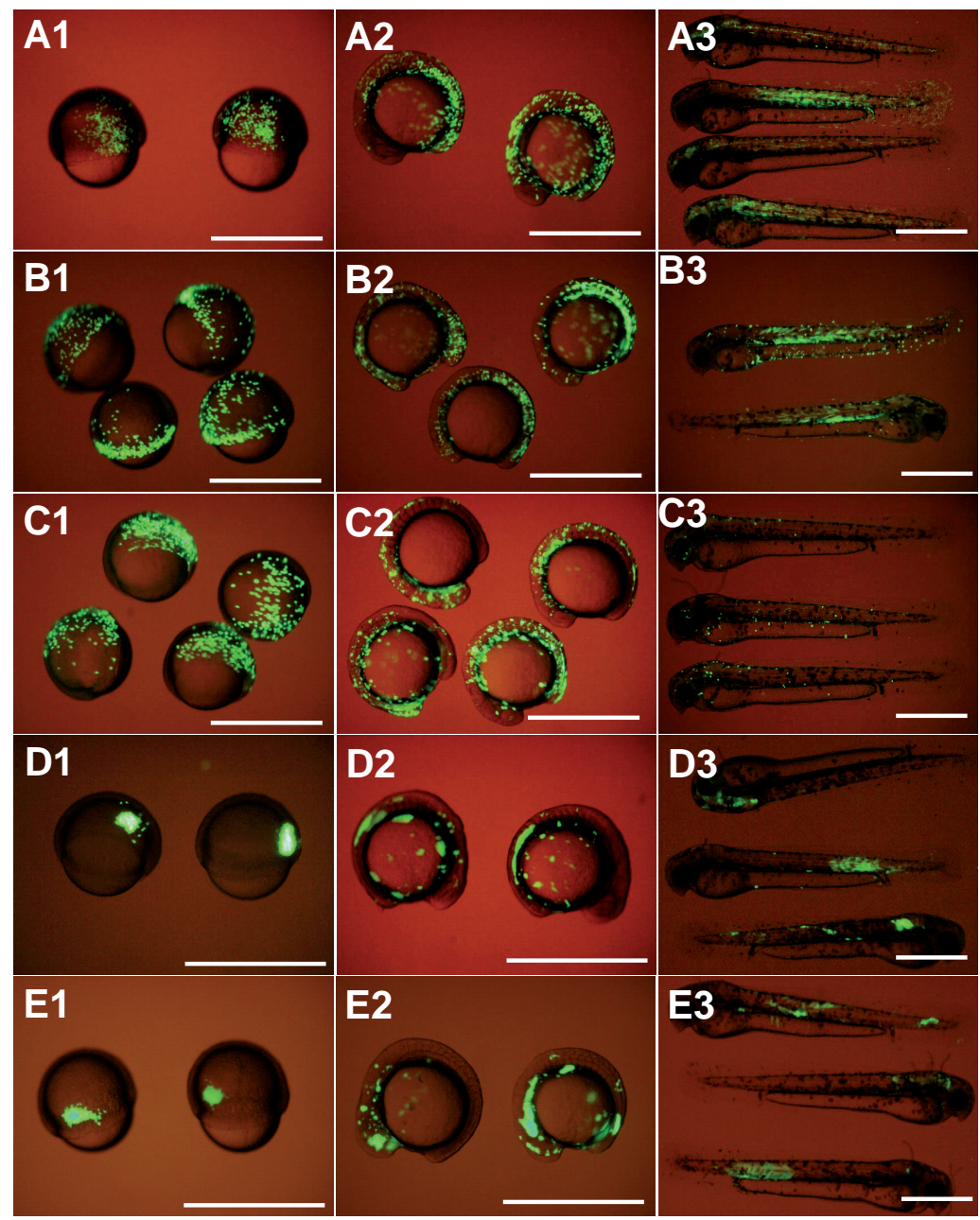

Fig. 3. Distribution of donor cells in chimeras using loach embryos as recipients. Green fluorescent cells indicate donor cells. Donor cells derived from diploid loach (A), hybrid (loach eggs $\times$ goldfish sperm) (B), the nucleocytoplasmic hybrid (C), diploid goldfish (D), hybrid (goldfish eggs $\times$ loach sperm) (E). Column 1, gastrula stage. Column 2, somitogenesis stage. Column 3, hatching stage. Scale bars indicate $1 \mathrm{~mm}$.

difference suggests that nucleocytoplasmic incompatibilities might be revealed more prominently in haploid nucleocytoplasmic hybrids. We therefore suggest that our experimental system, i.e. haploid nucleocytoplasmic hybrids, will be of value for elucidating the interactions between the nucleus and cytoplasm.

Our nucleocytoplasmic hybrid embryos developed normally during the initial cleavage period, but their subsequent development arrested at the dome stage, and they did not form gastrulalike structures. In comparison, the other types of embryos that we constructed, with the exception of the anuclear embryos, essentially completed the gastrulation process in a normal fashion. Developmental arrest at an early stage of embryogenesis has also been reported in amphibian nucleocytoplasmic hybrids (reviewed in Gallien, 1979). Nucleocytoplasmic hybrids formed using Rana palustris nuclei and $R$. pipiens cytoplasm had irreversible chromosome aberrations (Hennen, 1963). Similarly in teleosts, the uniparental elimination of chromosomes and subsequent occurrence of aneuploidies have been reported in several combinations of interspecific inviable salmonid hybrids (Arai, 1984; Fujiwara et al., 1997) and Oryzias hybrids (Iwamatsu et al., 2003; Sakai et al., 2007). However, diploid hybrids between male goldfish and female loach, which have the same combination of paternally-derived nucleus and egg cytoplasm as our nucleocytoplasmic hybrids, developed abnormally and died after hatching as previously reported by Suzuki (1953). These latter hybrids have an intermediate karyotype, comprising haploid sets derived from both species (Kijima et al., 1996). Nuclear-transplant nucleocytoplasmic hybrids having a diploid goldfish nucleus in egg cytoplasm of the largescale loach (Paramisgurnus dabryanus) do not exhibit chromosome elimination and aneuploidy in embryos (Yan et al., 1990). Thus, we presume that the developmental arrest in our nucleocytoplasmic hybrids could not be attributed to chromosome aberrations during embryogenesis.

We showed here that expression of the gsc and $n t /$ genes was aberrant in nucleocytoplasmic hybrids, suggesting a possible explanation for the developmental arrest at the late blastula stage. The initial timing and spatial patterns of $g s c$ and $n t /$ expression in nucleocytoplasmic hybrid embryos were similar to those of the control embryos. Zygotic expression of $g s c$ and $n t /$ are induced by maternally derived signals from the yolk cell and the developmental fate of blastomeres is specified by expression of these genes (Schulte-Merker et al., 1992; Stachel et al., 1993; Mizuno et al., 1996; Ober and Schulte-Merker, 1999). $g s c$ and $n t /$ expressions in the nucleocytoplasmic hybrid at the late blastula stage indicates that the haploid goldfish genome is likely controlled by the maternally-derived loach egg cytoplasm. The expression of these genes in nucleocytoplasmic hybrids, however, was not maintained throughout early embryonic development, and their signals finally disappeared at the time when diploid loach embryos had reached the $100 \%$ epiboly stage. After the late blastula stage, the interactions of various signaling pathways are presumed to play important roles in morphogenetic movement, such as convergence and extension, during gastrulation (Schier and Talbot, 2005). In our nucleocytoplasmic hybrid, the embryogenesis resulted in the disappearance of signals from both genes and the consequent lack of morphogenetic movements. This result implies that the nucleocytoplasmic incompatibility induces unsustainable signaling pathways.

In the present study, we produced chimeras by transplantation of blastomeres derived from nucleocytoplasmic hybrids into loach or goldfish embryos. The chimeras were then analyzed to assess the viability and the characteristics of the blastomeres in the recipient embryos. Analysis of chimeras is a powerful tool for verifying the developmental potential of embryonic cells derived from lethal embryos. In amphibians, when embryonic cells derived from lethal haploids are transplanted into viable diploid embryos to produce haploid-diploid chimeras, the transplanted cells are able to survive and differentiate (Hamilton, 1963, Kashiwagi and Kashiwagi, 1993). It was also reported that cells from the embryonic lethal hybrid between an $R$. pipiens female $\times$ $R$. sy/vatica male could be rescued by transplantation into fully 
viable recipient embryos (Moore, 1947). Recently, haploid-diploid chimeras were experimentally produced by transplantation of blastoderm using goldfish embryos (Tanaka et al., 2004). The transplanted haploid cells were detected in some organs of the diploid embryos and the resultant haploid-diploid chimeras survived to adulthood (Tanaka et al., 2004).

In our experiment, ten different types of chimera were produced by various combinations of five types of donor embryo and two types of recipient embryo (Table 2). The behavior of donor cells in each chimeric combination is summarized in Table 3. We found that the distribution of donor cells in the chimeras depended on whether the cytoplasm of the donor and recipient species was matched or not. When an intraspecific combination of donor and recipient was used, the donor cells mingled with those of the recipient in the chimeric embryos during the gastrula stage, and were then widely distributed throughout the hatching larvae. However, when an interspecific combination of donor and recipient was used, which had an unmatched cytoplasmic combination, we observed aggregations of the donor cells in the chimeric embryos. Donor cells from nucleocytoplasmic hybrids were seen to mingle with those of the loach embryo recipients; in this instance, the donor and recipient were of the same cytoplasmic origin. In contrast, donor cells from nucleocytoplasmic hybrids
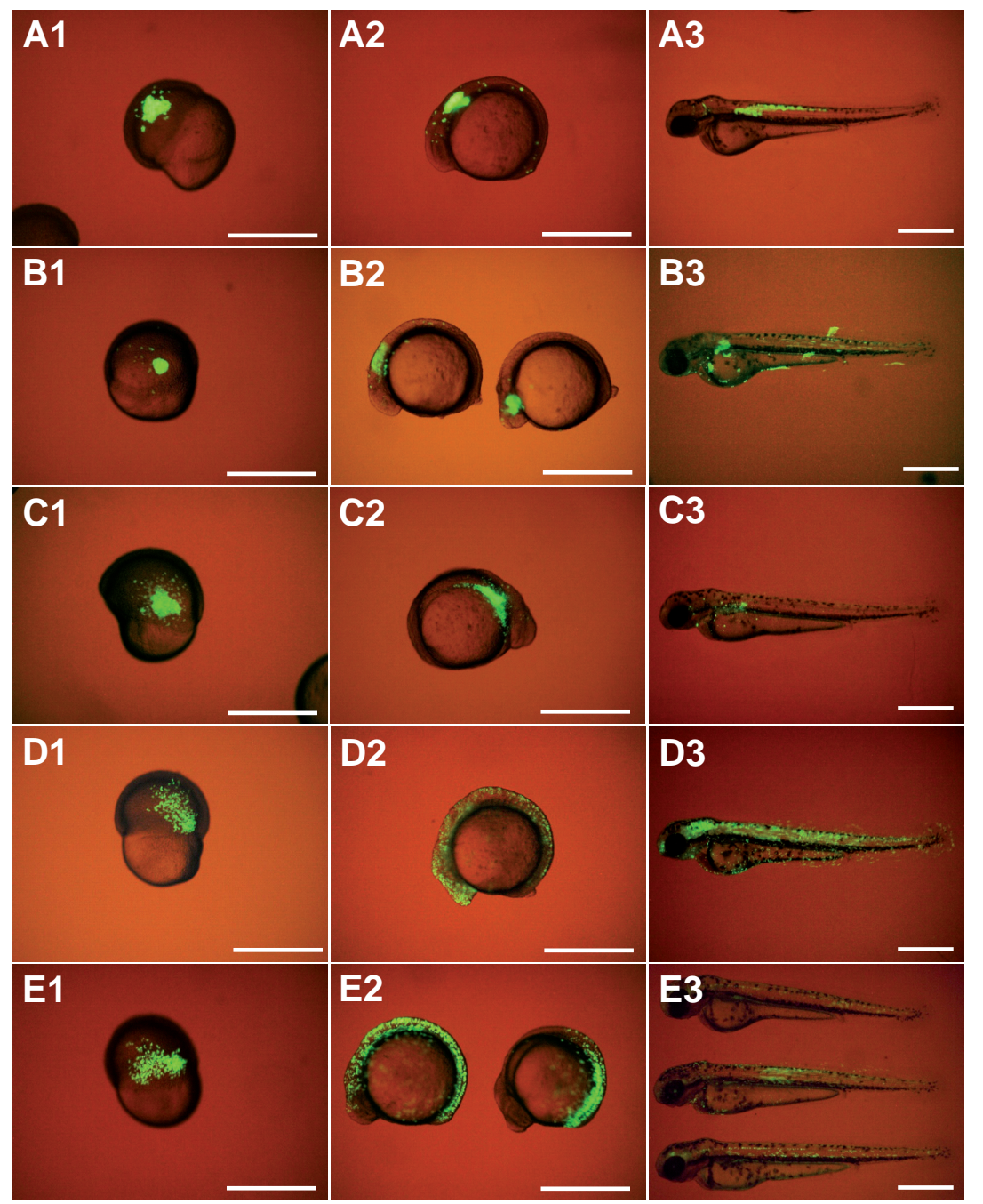

TABLE 1

\section{COMBINATIONS OF EGGS AND SPERM USED TO GENERATE DIPLOID LOACH, HYBRID, GYNOGENETIC HAPLOID LOACH, ANDROGENETIC HAPLOID LOACH, NUCLEOCYTOPLASMIC HYBRID AND ANUCLEAR EMBRYOS}

\begin{tabular}{|c|c|c|c|}
\hline & Eggs & Sperm & $\begin{array}{l}\text { Observation points in development and gene } \\
\text { expression during gastrulation period }\end{array}$ \\
\hline Diploid loach & Loach & Loach & $\begin{array}{l}\text { Intact control group: } \\
\text { Standard development and gene expression } \\
\text { under diploid loach genome }\end{array}$ \\
\hline Hybrid & Loach & Goldfish & $\begin{array}{l}\text { Control group: } \\
\text { Effects of hybrid nucleus comprising loach and } \\
\text { goldfish genome with loach cytoplasm }\end{array}$ \\
\hline $\begin{array}{l}\text { Gynogenetic } \\
\text { haploid loach }\end{array}$ & Loach & UV goldfish & $\begin{array}{l}\text { Control group: } \\
\text { Effects of haploid loach nucleus }\end{array}$ \\
\hline $\begin{array}{l}\text { Androgenetic } \\
\text { haploid loach }\end{array}$ & UV loach & Loach & $\begin{array}{l}\text { Control group: } \\
\text { Effects of UV irradiation on loach cytoplasm }\end{array}$ \\
\hline $\begin{array}{l}\text { Nuceocytoplasmic } \\
\text { hybrid }\end{array}$ & UV loach & Goldfish & $\begin{array}{l}\text { Experimental group: } \\
\text { Nucleocytoplasmic interaction between goldfish } \\
\text { genome and loach cytoplasm }\end{array}$ \\
\hline Anuclear & UV loach & UV goldfish & $\begin{array}{l}\text { Control group: } \\
\text { Effects of cytoplasmic factor without any } \\
\text { genomic contribution }\end{array}$ \\
\hline
\end{tabular}

formed aggregates in chimeras using goldfish embryos as recipients. In this instance, the donor and the recipient had cytoplasm that originated from different species. These results suggest that the species origin of donor cells is recognized in blastomeres at the gastrula stage, not by their nuclear genome but by their cytoplasm, despite the fact that transcription from the zygotic genome has already commenced by this stage.

Hennen (1973) produced chimeras in which donor grafts with diploid Rana palustris nuclei in $R$. pipiens cytoplasm were transplanted into recipient $R$. palustris embryos. These transplanted grafts survived for several days but failed to differentiate. In the chimeric embryos analyzed here, the donor cells from nucleocytoplasmic hybrids were also difficult to differentiate into various tissues. Furthermore, the population of donor cells from nucleocytoplasmic hybrids fell drastically during the somitogenesis to hatching stages, although the transplanted donor cells had successfully survived or mingled with recipient cells at the gastrula stage. Presumably, the major portions of cells in the nucleocytoplasmic hybrid do not have ability to differentiate and survive in the recipient embryos. On the other hand, such disruption was not observed in the other types of donor cells which formed clusters in the recipient embryos during embryogenesis. Thus, we concluded that the embryonic cells of the nucleocytoplasmic hybrid died due to nucleocytoplasmic incompatibility. However, a few donor cells from the nucleocyto-

Fig. 4. Distribution of donor cells in chimeras using goldfish embryos as recipients. Green fluorescent cells indicate donor cells. Donor cells derived from diploid loach (A), hybrid (loach eggs $\times$ goldfish sperm) (B), the nucleocytoplasmic hybrid (C), diploid goldfish (D), hybrid (goldfish eggs $\times$ loach sperm) (E). Column 1, gastrula stage. Column 2, somitogenesis stage. Column 3, hatching stage. Scale bars indicate $1 \mathrm{~mm}$. 
TABLE 2

\section{VARIOUS COMBINATIONS OF DONOR CELLS AND RECIPIENT EMBRYOS USED TO GENERATE THE DIFFERENT TYPES OF CHIMERA ANALYZED IN THE PRESENT STUDY}

\begin{tabular}{|c|c|c|c|}
\hline \multirow{2}{*}{$\begin{array}{l}\text { Species of } \\
\text { recipient embryo }\end{array}$} & \multirow{2}{*}{$\begin{array}{l}\text { Combination of female } \\
\text { and male in donor (female } x \text { male) }\end{array}$} & \multicolumn{2}{|c|}{$\begin{array}{c}\text { Nucleocytoplasmic } \\
\text { combination in the donor cell }\end{array}$} \\
\hline & & Cytoplasm & Nucleus (Ploidy) \\
\hline Loach & Loach x Loach & Loach & Loach (2n) \\
\hline Loach & Loach x Goldfish & Loach & Hybrid $^{* 1}(2 n)$ \\
\hline Loach & UV Loach x Goldfish & Loach & Goldfish (1n) \\
\hline Loach & Goldfish x Goldfish & Goldfish & Goldfish (2n) \\
\hline Loach & Goldfish x Loach & Goldfish & Hybrid $^{* 2}(2 n)$ \\
\hline Goldfish & Loach x Loach & Loach & Loach (2n) \\
\hline Goldfish & Loach x Goldfish & Loach & Hybrid $^{* 1}(2 n)$ \\
\hline Goldfish & UV Loach x Goldfish & Loach & Goldfish (1n) \\
\hline Goldfish & Goldfish x Goldfish & Goldfish & Goldfish (2n) \\
\hline Goldfish & Goldfish x Loach & Goldfish & Hybrid $^{* 2}(2 n)$ \\
\hline
\end{tabular}

"1: Hybrid between loach female and goldfish male.

"2: Hybrid between goldfish female and loach male.

plasmic hybrid existed in the recipient embryos. These cells might have viability and differential ability.

In conclusion, although the development of nucleocytoplasmic hybrids was arrested at a dome-like stage and they died at a time corresponding to the end of the gastrula stage in diploid loach embryos, embryonic cells did survive in chimeric embryos beyond the somitogenesis stage. This result suggests that embryonic cells of lethal nucleocytoplasmic hybrids can survive depending on the cytoplasmic environment in the recipient embryo.

\section{Materials and Methods}

\section{Collection of gametes, fertilization of eggs, and incubation of zy- gotes}

Loach (Misgurnus anguillicaudatus) and goldfish (Carassius auratus) were reared at the Nanae Fresh-Water Laboratory, Field Science Center for Northern Biosphere, Hokkaido University. The collection of gametes and the fertilization of eggs were performed as previously described (loach: Fujimoto et al., 2004; goldfish: Yamaha and Yamazaki, 1993). In the following experiments, fertilized eggs were dechorionated with Ringer's solution (128 mM NaCl, $2.8 \mathrm{mM} \mathrm{KCl}$ and $1.8 \mathrm{mM} \mathrm{CaCl}_{2}$ ) containing $0.1 \%$ trypsin and $0.4 \%$ urea, and transferred to a petri dish coated with $1 \%$ agar and filled with the culture medium \#1 (Ringer's solution containing 1.6\% albumen). Developmental stages of the resulting embryos were determined as described by Fujimoto et al. (2006).

\section{Genetic inactivation of gametes and the induction of nucleocyto- plasmic hybrids}

Loach eggs and goldfish sperm were genetically inactivated by irradiating with 150 or $50 \mathrm{~mJ} / \mathrm{cm}^{2} \mathrm{UV}$, respectively, as described previously (Fujimoto et al., 2007). Nucleocytoplasmic hybrids were then produced along with various combinations of embryos (Table 1) described below in order to compare developmental potentials with them. Nucleocytoplasmic hybrids were produced by fertilizing UV-irradiated loach eggs with a haploid sperm from a goldfish (UV loach eggs $\times$ goldfish sperm). Normal diploid loach embryos were produced by normal fertilization (loach eggs $\times$ loach sperm). Interspecific hybrid embryos were produced by interspecific fertilization (loach eggs $\times$ goldfish sperm). Gynogenetic haploid embryos were induced by fertilizing normal loach eggs with UV-irradiated goldfish sperm (loach eggs $\times$ UV goldfish sperm). Androgenetic haploid embryos were produced by fertilizing UV-irradiated loach eggs with loach
TABLE 3

\section{DISTRIBUTION OF DONOR CELLS IN CHIMERIC EMBRYOS AT THE GASTRULA STAGE, THE SOMITOGENESIS STAGE AND THE HATCHING STAGE}

\begin{tabular}{|c|c|c|c|c|}
\hline \multicolumn{2}{|r|}{ Chimera } & \multicolumn{3}{|c|}{ Developmental stages } \\
\hline Recipient & Donor (female x male) & Gastrula & Somitogenesis & Hatching \\
\hline Loach & Loach x Loach & intermingled & scattered & scattered \\
\hline Loach & Loach x Goldish & intermingled & scattered & scattered \\
\hline Loach & UV Loach x Goldfish & intermingled & clustered ${ }^{*}$ & spotty \\
\hline Loach & Goldfish x Goldfish & aggregated & clustered & clustered \\
\hline Loach & Goldfish x Loach & aggregated & clustered & clustered \\
\hline Goldfish & Loach x Loach & aggregated & clustered & clustered \\
\hline Goldfish & Loach x Goldish & aggregated & clustered & clustered \\
\hline Goldfish & UV Loach x Goldfish & aggregated & clustered & spotty \\
\hline Goldfish & Goldfish x Goldfish & intermingled & scattered & scattered \\
\hline Goldfish & Goldfish x Loach & intermingled & scattered & scattered \\
\hline
\end{tabular}

: The donor cells form small clusters in the chimeric embryos.

sperm (UV loach eggs $\times$ loach sperm). Anuclear embryos were generated by fertilizing UV-irradiated loach eggs with UV-irradiated goldfish sperm (UV loach eggs $\times$ UV goldfish sperm). In each experiment, gametes were collected from a single female and male. Each experimental group was simultaneously generated with the same egg batch and sperm from goldfish and loach.

\section{Whole mount in situ hybridization}

Whole mount in situhybridization was used to examine the expression patterns of the goosecoid (gsc)and no tail (ntl) genes in the various types of embryo described above. The embryos were fixed with $4 \%$ paraformaldehyde in phosphate buffered saline for $30 \mathrm{~h}$ when the normal diploid loach embryos reached the dome, germ ring, $75 \%$ epiboly and $100 \%$ epiboly stages, and were stored in $100 \%$ methanol at $-20^{\circ} \mathrm{C}$.

Expression of $g s c$ was detected with an antisense digoxigeninlabeled gsc RNA probe transcribed from an approximately $0.4 \mathrm{~Kb}$ fragment excluded the homeodomain of zebrafish gsccDNA (Stachel et al., 1993). Expression of $n t /$ was detected with an antisense fluoresceinlabeled $n t /$ RNA probe transcribed from an approximately $1.8 \mathrm{~Kb}$ fragment without the 3' UTR region of zebrafish $n t /$ cDNA (SchulteMerker et al., 1992). These probes derived from zebrafish gene sequences were used for detection of $g s c$ and $n t /$ transcripts in goldfish and loach embryos (Mizuno et al., 1997; Fujimoto et al., 2004). These probes were generated by in vitrotranscription according to the manufacturer's instructions (Roche). Hybridization, washing and detection steps in whole mount in situ hybridization were carried out as previously described (Mizuno et al., 1997; Fujimoto et al., 2006).

\section{Induction of chimeras}

Blastomeres from nucleocytoplasmic hybrids and other types of embryos, such as diploid loach, diploid goldfish and reciprocal hybrids (loach eggs $\times$ goldfish sperm, goldfish eggs $\times$ loach sperm) were transplanted into diploid loach and diploid goldfish embryos; the recipient embryos were at the oblong to sphere stages. Consequently, five types of donor and two types of recipient were used to induce chimeras. The various combinations of donor cells and recipient embryos are summarized in Table 2.

In order to monitor the behavior of transplanted blastomeres, donor embryos at the one-cell to four-cell stages were labeled by microinjection just under the blastoderm of $2.5 \%$ fluorescein isothiocyanate (FITC)dextran (average mol. wt. 10,000; Sigma) diluted in $0.2 \mathrm{M} \mathrm{KCl}$. The injected embryos were then incubated in culture medium \#1 (see above). Unfertilized eggs and unlabelled embryos were removed prior to chimera production at the blastula stage. Transplantation of blastomeres for induction of chimeras was carried out when the donor and recipient 
embryos had reached the oblong to dome stages. The blastomeres used for transplantation were obtained by gentle aspiration of the outer part of the donor blastoderm using a glass micropipette with an $80-100 \mu \mathrm{m}$ bore diameter connected to an injector (CellTram vario; Eppendorf). The donor blastomeres were transplanted into the inner side margin of the recipient blastoderm without dissociation of the graft. The embryos were incubated in culture medium \#1 until the $100 \%$ epiboly stage and then in culture medium \#2 (1.8 mM MgCl $2,1.8 \mathrm{mM} \mathrm{CaCl}_{2}, 0.01 \%$ penicillin and $0.01 \%$ streptomycin) until the hatching stage. The distribution of donor cells in chimeric embryos was determined using a fluorescence microscope (MZ12; Olympus) at the gastrula (50\% to $70 \%$ epiboly), somitogenesis (before yolk extension) and hatching (one day after hatching) stages.

We classified behaviors of donor cells in each type of chimera into "intermingled", "aggregated", "scattered", "clustered" and "spotty". When most part of donor cells were discretely dissociated in recipient embryo at the gastrula stage, the status of donor cells was classified into "intermingled". In contrast, "aggregated" was applied to the occasion, when most part of donor cells formed a cell mass adhering to each other at the gastrula stage. At the somitogenesis and the hatching stage, "clustered" was applied when donor cells formed some aggregations in recipient embryos. On the other hand, "scattered" was defined when donor cells were distributed in recipient embryos without any unusual cell mass consisted of donor cells. In hatching stage, "spotty" were dotted with a few donor cells in hatching larvae.

\section{Acknowledgement}

This study was supported in part by a Grant-in-Aid for the 21st Century COE program (K-02, 2006-2008) for Graduate School of Fisheries Sciences of Hokkaido University from the Ministry of Education, Culture, Sports, Science and Technology (MEXT), Japan, that for Scientific Research (B) from Japan Society for the Promotion of Science (JSPS) to E. Yamaha (No. 16380124) and that for Promotion of Basic Research Activities for Innovative Biosciences (PROBRAIN).

\section{Reference}

ARAI, K. (1984). Developmental genetic studies on salmonids: morphogenesis, isozyme phenotype and chromosomes in hybrid embryos. Mem Fac Fish Hokkaido, Univ. 31: 1-94.

BERCSÉNYI, M., MAGYARY, I., URBÁNYI, B., ORBÁN, L. and HORVÁTH, L. (1998). Hatching out goldfish from common carp eggs: interspecific androgenesis between two cyprinid species. Genome 41: 573-579.

CORLEY-SMITH, G.E. and BRANDHORST, B.P. (1999). Preservation of endangered species and populations: A role for genome banking, somatic cell cloning, and androgenesis? Mol Reprod Dev 53: 363-367.

FUJIMOTO, T., KATAOKA, T., OTANI, S., SAITO, T., AITA, T., YAMAHA, E. and ARAI, K. (2004). Embryonic stages from cleavage to gastrula in the loach Misgurnus anguillicaudatus. Zool Sci21: 747-755.

FUJIMOTO, T., KATAOKA, T., SAKAO, S., SAITO, T., YAMAHA, E. and ARAI, K. (2006). Developmental stages and germ cell lineage of the loach (Misgurnus anguillicaudatus). Zool Sci23: 977-989.

FUJIMOTO, T., SAKAO, S., YAMAHA, E. and ARAI, K. (2007). Evaluation of different doses of UV irradiation to loach eggs for genetic inactivation of the maternal genome. J EXp Zoo/307A: 449-462.

FUJIWARA, A., ABE, S., YAMAHA, E., YAMAZAKI, F. and YOSHIDA, M.C. (1997). Uniparental chromosome elimination in the early embryogenesis of the inviable salmonid hybrids between masu salmon female and rainbow trout male. Chromosoma 106: 44-52.

GALLIEN, C.L. (1979). Expression of nuclear and cytoplasmic factors in ontogenesis of amphibian nucleocytoplasmic hybrids. Int Rev Cytol Supp/9: 189-219.

HAMILTON, L. (1963). An experimental analysis of the development of the haploid syndrome in embryos of Xenopus laevis. J Embryo Exp Morph 11: 267-278.

HENNEN, S. (1963). Chromosomal and embryological analyses of nuclear changes occurring in embryos drived from transfers of nuclei between Rana pipiens and
Rana sylvatica. Dev Bio/6: 133-183.

HENNEN, S. (1965). Nucleocytoplasmic hybrids between Rana pipiens and Rana palustris. I. Analysis of the developmental properties of the nuclei by means of nuclear transplantation. Dev Bio/11: 243-267.

HENNEN, S. (1973). Competence tests of early amphibian gastrula tissue containing nuclei of one species (Rana palustris) and cytoplasm of another (Rana pipiens). J Embryo Exp Morph 29: 529-538.

IWAMATSU, T., KOBAYASHI, H., YAMASHITA, M., SHIBATA, Y. and YUSA, A. (2003). Experimental hybridization among Oryzias species. II. Karyogamy and abnormality of chromosome separation in cleavage of interspecific hybrids between Oryzias latipes and O. Javanicus. Zool Sci20: 1381-1387.

KASHIWAGI, K. and KASHIWAGI, A. (1993). Artificially produced chimeras between haploids and diploids in Japanese anurans. Sci Rep Lab Amphibian Biol, Hiroshima Univ 12: 37-53.

KIJIMA, K., ARAI, K., SUZUKI, R. (1996). Induced allotriploidy in inviable interfamilial hybrids, female loach $\mathrm{x}$ male goldfish and female loach $\mathrm{x}$ male minnow. $J$ Fac Appl Biol Sci, Hiroshima Univ 35: 1-12.

KOMEN, H. and THORGAARD, G.H. (2007). Androgenesis, gynogenesis and the production of clones in fishes: A review. Aquaculture 269: 150-173.

MIZUNO, T., YAMAHA, E., WAKAHARA, M., KUROIWA, A. and TAKEDA, H. (1996). Mesoderm induction in zebrafish. Nature 383: 131-132.

MIZUNO, T., YAMAHA, E. and YAMAZAKI, F. (1997). Localized axis determinant in the early cleavage embryo of the goldfish, Carassius auratus. Dev Genes Evol 206: 389-396.

MOORE, J.A. (1947). Studies in the development of frog hybrids. II. Competence of the gastrula ectoderm of Rana pipiens $q \times$ Rana sylvatica o hybrids. J Exp Zool105: 349-370.

NAM, Y.K., CHO, Y.S., CHO, H.J. and KIM, D.S. (2002). Accelarated growth performance and stable germ-line transmission in androgenetically derived homozygous transgenic mud loach, Misgurnus mizolepis. Aquaculture 209: 257-270.

NAM, Y.K., CHO, Y.S. and KIM, D.S. (2000). Isogenic transgenic homozygous fish induced by artificial parthenogenesis. Transgenic Res 9: 463-469.

OBER, E.A. and SCHULTE-MERKER, S. (1999). Signals from the yolk cell induce mesoderm, neuroectoderm, the trunk organizer, and the notochord in zebrafish. Dev Bio/215: 167-181.

SAKAI, C., KONNO, F., O.NAKANO, IWAI, T., YOKOTA, T., LEE, J., NISHIDAUMEHARA, C., KUROIWA, A., MATSUDA, Y. and YAMASHITA, M. (2007). Chromosome elimination in the interspecific hybrid medaka between Oryzias latipes and O. hubbsi. Chromosome Res 15: 697-709.

SCHIER, A.F. and TALBOT, W.S. (2005). Molecular genetics of Axis formation in zebrafish. Annu Rev Genet 39: 561-613.

SCHULTE-MERKER, S., HO, R.K., HERRMANN, B.G. and NÜSSLEIN-VOLHARD, C. (1992). The protein product of the zebrafish homologue of the mouse Tgene is expressed in nuclei of the germ ring and the notochord of the early embryo. Development 116: 1021-1032.

STACHEL, S.E., GRUNWALD, D.J. and MYERS, P.Z. (1993). Lithium perturbation and goosecoid expression identify a dorsal specification pathway in the pregastrula zebrafish. Development 117: 1261-1274.

SUZUKI, R. (1953). Studies on the interfamiliar crossing between loach (Misgurnus anguillicaudatus) and goldfish (Carassius auratus). Jpn J Ichthyo/3: 7-14.

TANAKA, M., YAMAHA, E. and ARAI, K. (2004). Survival capacity of haploid-diploid goldfish chimeras. J EXp Zoo/301A: 491-501.

TUNG, T.C. and TUNG, Y.F.Y. (1963). Nuclear transfer in vertebrates. Acta Zool $\operatorname{Sin}$ 15: 151-167.

TUNG, T.C., TUNG, Y.F.Y., LUH, T.Y., TUNG, S.M. and TU, M. (1973). Transplantation of nuclei between two subfamilies of teleosts (goldfish-domesticated Carassius auratus, and Chinese bitterling-Rhodeus sinensis). Acta Zoo/Sin19: 201-212.

WAKAMATSU, Y., JU, B., PRISTYAZNHYUK, I., NIWA, K., LADYGINA, T., KINOSHITA, M., ARAKI, K. and OZATO, K. (2001). Fertile and diploid nuclear transplants derived from embryonic cells of a small laboratory fish, medaka (Oryzias latipes). Proc Nat/ Acad Sci USA 98: 1071-1076.

YAMAHA, E. and YAMAZAKI, F. (1993). Electrically fused-egg induction and its 
development in the goldfish, Carassius auratus. Int J Dev Bio/37: 291-298.

YAN, S.Y. (1989). The nucleo-cytoplasmic interactions as revealed by nuclear transplantation in fish. In Cytoplasmic organization systems, vol. 5 (ed. MALACINSKI, G. M.). McGraw-Hill, New York, pp.61-81.
YAN, S.Y., TU, M., YANG, H.Y., MAO, Z.R., ZHAO, Z.Y., FU, L.J., LI, G.S., HUANG, G.P., LI, S.H., JIN, G.Q. et al. (1990). Developmental incompatibility between cell nucleus and cytoplasm as revealed by nuclear transplantation experiments in teleost of different families and orders. Int J Dev Bio/35: 255-265.

\section{Further Related Reading, published previously in the Int. J. Dev. Biol.}

See Special Issue Pattern Formation edited by Michael K. Richardson and Cheng-Ming Chuong at: http://www.ijdb.ehu.es/web/contents.php?vol=53\&issue=5-6

The formation of primordial germ cells from germline cells in spherical embryos derived from the blastodisc of 2-cell embryos in goldfish, Carassius auratus

Satoshi Otani, Tomoe Kitauchi, Taiju Saito, Suzu Sakao, Shingo Maegawa, Kunio Inoue, Katsutoshi Arai and Etsuro Yamaha Int. J. Dev. Biol. (2005) 49: 843-850

Effect of «decreasing of sensitivity» to heparin of casein kinase II type (CK II) in early development of loach Misgurnus fossilis L E A Zemskov and E B Abramova Int. J. Dev. Biol. (1996) 40: S235-S236

Electrically fused-egg induction and its development in the goldfish, Carassius auratus E Yamaha and F Yamazaki Int. J. Dev. Biol. (1993) 37: 291-298

Mitochondrial DNA content and mitochondrial gene transcriptional activities in the early development of loach and goldfish

G Wang and S Yan

Int. J. Dev. Biol. (1992) 36: 477-482

Further investigation on nuclear transplantation in different orders of teleost: the combination of the nucleus of Tilapia (Oreochromis nilotica) and the cytoplasm of Loach (Paramisgurnus dabryanus)

S Y Yan, Z R Mao, H Y Yang, M A Tu, S H Li, G P Huang, G S Li, L Guo, G Q Jin and R F He Int. J. Dev. Biol. (1991) 35: 429-435

Surface polarization in loach eggs and two-cell embryos: correlations between surface relief, endocytosis and cortex contractility

$\mathrm{V} V$ Ivanenkov, V N Meshcheryakov and L E Martynova

Int. J. Dev. Biol. (1990) 34: 337-349

Developmental incompatibility between cell nucleus and cytoplasm as revealed by nuclear transplantation experiments in teleost of different families and orders S Y Yan, M Tu, H Y Yang, Z G Mao, Z Y Zhao, L J Fu, G S Li, G P Huang, S H Li and G Q Jin Int. J. Dev. Biol. (1990) 34: 255-266

Fibronectin-rich fibrillar extracellular matrix controls cell migration during amphibian gastrulation

J C Boucaut, K E Johnson, T Darribère, D L Shi, J F Riou, H B Bache and M Delarue Int. J. Dev. Biol. (1990) 34: 139-147

5 yr ISI Impact Factor $(2008)=3.271$

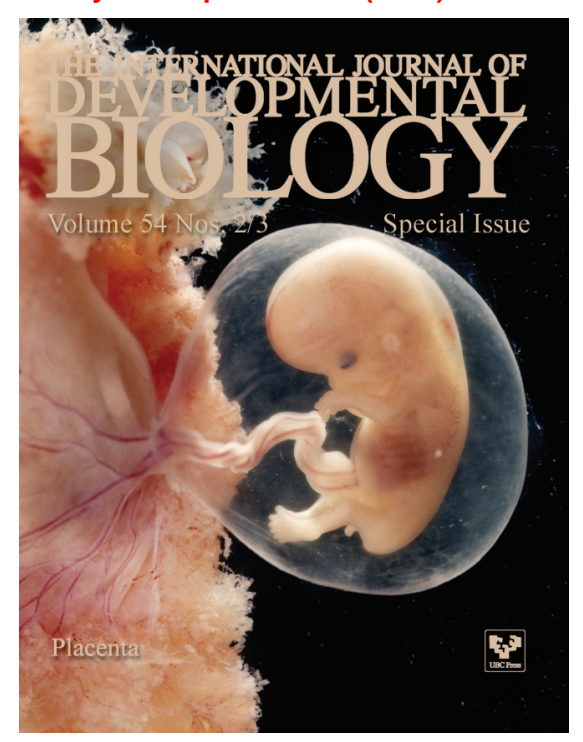

